\title{
Author postprint
}

Accepted for publication in Children Australia

Title: Pre-placement planning: Examining continuity and connections

Authors:

Clare Tilbury, Life Without Barriers Carol Peltola Research Chair

Jennifer Osmond, Adjunct Senior Lecturer

Affiliation: School of Human Services and Social Work, Griffith University, Australia

Keywords: placement planning, matching, stability, continuity, foster care

Acknowledgement: This research was supported by Life Without Barriers. 


\title{
Pre-placement planning: Examining continuity and connections
}

\begin{abstract}
It is well-recognised that children and young people in out-of-home care require stability and a sense of security. Focus has been given to the importance of stable placements with the same carers and maintaining or establishing positive relationships with significant others, such as parents and siblings. Undoubtedly, placement and family relationships are critical, but there are additional avenues for enhancing children's wellbeing when planning for continuity and connections. This paper reports on how continuity and connections for children in out-of-home care are dealt with in the placement planning process. Sixteen case managers were interviewed from a non-government placement service in two Australian states about pre-placement planning, connections, continuity and discontinuity. The findings suggest that continuity and connection planning should be a more elevated and explicit part of the process of pre-placement planning, as it has the potential to create a more seamless and coherent experience for children.
\end{abstract}


This paper focuses on pre-placement planning, with specific attention to the nature and level of continuity and connections that are sought when children commence or change placements that are arranged for their care and protection. 'Pre-placement' covers the period from a child requiring a first or new placement to the transition to the placement, and includes the information sought from, and provided to, the child, parents and potential foster or kinship carer. It includes identifying potential placements; the matching process; and planning moves from one placement to the next. Research exploring placement stability, placement breakdown and carer retention has established the importance of pre-placement planning (e.g. Berridge, 2000; Brown \& Campbell, 2007; Gilbertson \& Barber, 2003). Sensitively managed matching and introductions are noted as factors in successful shorter-term placements (Sellick \& Thoburn, 1996; Waterhouse \& Brocklesby, 2001). But overall, the literature on pre-placement planning is sparse, with the literature on matching concentrating on characteristics to match, such as ethnicity, locality, siblings, and age of other children in the foster home. It has been noted that the capacity to match is undermined if placement choice is limited, with 'best available' being displaced by 'first available' (Triseliotis et al., 2000). This was referred to as 'compromised matching' by Poso and Laakso (2014), to describe the navigation that occurs between professional principles, the views of children and their parents, and the social and economic conditions of placement agencies that can impact on placement quality when making placement decisions.

Continuity relates to something that is 'connected, unbroken and uninterrupted in time and sequence' (Fowler and Fowler, 1964, p. 264) Connection refers to 'the joining of two things together... associate mentally with, unite with others' (Fowler and Fowler, 1964, p. 257). Research outlines why continuity and connections are important for children in the out-of-home care system. One reason is that children in care may have already experienced considerable precare loss and discontinuity (Ficaro, 1999; Mudaly \& Tucci, 2002). While the decision to remove a child from home creates an intentional discontinuity of existing circumstances for the child (Van Nijnatten, 2010), entering out-of-home care heightens the risk that a child may experience other losses that can be detrimental to them. These losses include changes related to school, positive relationships, extracurricular activities, familiar routines, environment and places, neighbourhood, pets, and belongings. These discontinuities can have an impact on a child's development, identity formation, and long-term mental health (Ward, 2011). The link between identity and continuity is also made by Van Nijnatten (2010) who explains that our capacity to govern our life and make decisions, our agency, is influenced by our opportunity to construct psychological continuity. In situations where there is child maltreatment or significant family difficulties, a child's development of self-continuity can be compromised if parents or carers are not be able to facilitate their sense of control (Van Nijnatten, 2010). Further, Jack (2010) outlines how connections to places and spaces can influence identity development.

Relationship continuity has been recognised as important for children and it reflects the importance of minimising the likelihood of attachment related difficulties. Secure attachments for children are facilitated by consistent, predictable, positive and nurturing relationships (Howe et al., 2000). Arranging and supporting contact with parents, siblings and extended family members is one of the tangible ways policy and practice has operationalised the importance of relationship continuity. Research about young people who have transitioned from care has canvassed perceptions of stability and related issues reinforcing the importance of belonging to family and social networks (described as 'felt security') (Cashmore \& Paxman, 1996; Cashmore \& Paxman, 2006). 
Besides placement stability and relationships with carers and family members, other areas have been identified for building continuity and connections. Jackson (2002, p. 42) categorises stability into six areas: placement - being stable with long-term consistent carers; relationships having a network of consistent people in the child's life that remain continuous; education consistent and stable place of schooling with quality support; health care - being able to receive health care that is tailored to a child's full history and individualised needs; community - a child having the opportunity to stay connected with a neighbourhood and community; and personal identity - for children to be able to develop a positive sense of self, clear understanding of their own identity and culture. Positive, nurturing relationships with teachers and other school staff can assist when family or carer relationships are not optimal (Gilligan, 1998). Gilligan's (2008) study focuses on the importance of recreational and social activities, given that family and school can be stressful for children in care. He argued that active participation in 'spare time' activities can positively influence developmental trajectories and outcomes, and build resilience.

Research has also identified the potential for children to develop new relationships, interests and networks while placed away from their families, and the vital role of carers in encouraging children placed in their care to explore and develop their interests and hobbies (e.g. Gilligan, 2008; Happer et al., 2006). Fong et al. (2006) argued that continuity for children in care can be enhanced in areas such as school, extracurricula activities, interests and peers, which provide opportunities to create normalisation and support for children. In exploring whether continuity of activities could increase child wellbeing, they investigated carer awareness about children's previous activities and involvements, and found that many carers were not aware of what a child had been involved in prior to placement.

In Australia, as in other countries, there are increasing numbers of children in care due to concerns about abuse or neglect. In 2010-11, there were 37,648 children in out-of-home care (7.3 per 1000), with 11,613 children entering care during the year. Most children were placed in home-based care, with $44 \%$ foster care, $47 \%$ in kinship care and $2 \%$ in other forms of homebased care (Australian Institute of Health and Welfare, 2013). Most (55\%) children exited care to return home to their families within two years of entering care (Steering Committee for the Review of Government Service Provision, 2013). However, the remaining 45\% of children in longer-term care were likely to experience numerous placements. For children who exited care after 12 months or more, 52\% had experienced more than two placements (Steering Committee for the Review of Government Service Provision, 2013). Multiple placement changes can be detrimental to children in all aspects of their social and emotional wellbeing and future life chances (Ward, 2009). Reducing the number of children in care by ensuring out-of-home placement is used only when necessary, in combination with carefully planned and supported reunification are obviously essential to improving stability. However, on current trends, many children will experience a succession of short-term placements. The reality of the short-term nature of most placements, by design or otherwise, suggests the need to plan for achieving continuity and connections in children's lives through means other than striving for an elusive long-term placement with the same carers.

The aim of the present study was to contribute to knowledge about placement stability and inform policy and program responses to promoting continuity and connection across placement changes for children in out-of-home care. In doing so, it investigated whether and how a child's existing connections were taken into account in decisions about where a child would be best placed, and the factors (such as child's interests, local placement, resource availability) that 
supported or impeded a child's continuity and connections. No previous Australian research was located about pre-placement planning or matching.

\section{Method}

We conducted a qualitative study based on semi-structured interviews with placement case managers to identify processes and factors involved in pre-placement planning and the level of continuity in particular aspects of in children's lives. A sample of 16 case managers was purposively recruited for the study with the assistance of the partner organisation, a large nongovernment out-of-home care service provider. Criterion sampling (Dudley, 2011) was used to ensure that interviews were undertaken with case managers who were directly involved in organising placements for children, and who had organised a placement in the past six months that they could recall the details for. A case manager's role is to liaise with the statutory child protection agency, train and support carers, arrange placements, and provide and coordinate supports and services to children in out-of-home care, so they are the key placement decisionmakers. Emails were sent by an agency manager to case managers who met the criteria, inviting their participation, and the names of those who agreed were provided to researchers. The data were collected on a confidential basis with approval from the authors' human research ethics committee.

We focused on the connections children formed through participation in everyday activities, in order to avoid a narrow conceptualisation of stability defined by the number of placements a child experiences. A data collection instrument was designed for the interviews based on the research of Fong et al. (2006), which categorised children's activities into six domains of school, leisure, family, church, social and therapeutic. These domains were adapted (as a consequence of the literature review) as follows: school or work; leisure and social activities; contact with family members or significant others (including previous foster carers); religious and cultural activities; therapeutic activities; household routines (rules or routines about bedtime, meals, television and chores); and personal possessions. Questions focused on the nature and extent of pre-placement planning; the activity domains; and participants' views on continuity and building connections. Questions probed what children were involved in prior to placement and whether these activities continued or changed when the placement commenced. The interview was conducted by telephone and duration ranged from forty-five minutes to one hour. The interviewer took detailed notes of the participants' responses and these notes were transcribed for analysis.

During the data analysis phase, interview notes were carefully considered to identify the main themes or patterns, the common threads between the interviews (Miles \& Huberman, 1994). A manual, not computer-assisted method, was used to identify and analyse themes within the data. During the first stage of analysis, notes were made tentatively naming emerging conceptual ideas. These ideas were checked and reconsidered across all transcripts so all themes were recognised. The second stage involved grouping of themes and identification of any further subthemes, so both broad and more specific variations could be considered. These themes represent the integrating concepts that participants articulated as the important elements of pre-placement planning. Frequencies were calculated for the activity domains.

\section{Findings}

All participants were female, in the age range 20 to 55 years. All but one participant had educational qualifications in psychology, social work, human services, education, or similar field. Participants' experience in the human service industry ranged from 18 months to over 20 years. The 16 case managers discussed the placements of 17 children. The age range of the 
children and young people was 3 to 16 years, 10 children were female and 7 male. The majority of the children were Anglo-Australian ( $n=13), 1$ was Aboriginal, 3 were from other cultural backgrounds, and one child's cultural background was unknown.

Pre-placement planning

There was a high degree of commonality about what was considered to be pre-placement planning. It involved a series of steps and processes which aimed to heighten the likelihood of a successful match. The factors considered when undertaking pre-placement planning was influenced by agency guidelines, and reflected recognition that children may have multiple needs:

A planned process should involve nine dimensions: education, culture and spirituality, health and physical wellbeing, family contact, relationships, emotional wellbeing, recreation, safety and stability, living skills, transition from care, and legal needs (\#11) What information was obtained, and from whom? To identify a possible promising placement, a variety of information was sought: behaviour issues of the child, finding out about the medical, education, health, cultural and identity needs of the child. Other information that was gathered was background, referral information from statutory department, information from others (including from family members, previous case managers, services involved, and former carers). One participant pointed out that although information was gathered from others, it was important for her to be independent in her thinking about a placement.

Information from the child or young person. Many participants made particular reference to the importance and value of speaking directly with the child and gaining information from him or her about preferences and ideas for placement. However, a number of the participants commented that they felt that they really knew the children they were working with and did not need to undertake additional information gathering.

Who was information given to? Information that was gathered was shared with other people involved in making a decision about a possible placement (e.g. placement panels, senior staff) and also the potential carer. On informing carers about a child who may be placed with them one participant indicated that some censoring or restriction of information was at times necessary to ensure that carers did not become overwhelmed before the child arrived.

The process elements of pre-placement planning that were identified included: give information to the child about the placement they are going to; visit the placement; arrange a meet and greet between the child and the carer (this could be a picnic, visit, or sleepover); use a step-by-step process; provide time to try the placement; arrange home visits; undertake a possession inventory; and talk with the child and carer about household routines and rules. It was raised that opportunities should be created for children to ask lots of questions about the household, such as when they can have friends visit, meals and snacks, bedtime routine, pocket money, household routines and expectations. These processes were considered beneficial for both children and carer determining whether the placement would be a match and viable. A gradual and smooth transitional experience was considered ideal. Participants outlined the principles based on agency guidelines and from their experience that they considered underpinned their practice when placement planning. Practice principles that were identified by participants are summarised in Table 1.

Insert Table 1 here

Participants had a strong desire to find a placement that would prove successful. However, sometimes this meant that not all the principles could be adhered to due to availability of placements. Despite the limitations on placement choice, pre-placement planning was thought to 
increase the likelihood of meeting the child's needs and helping the child settle into a placement, so was important for enhancing stability. It helped to maintain connections to previous placements, created a smooth transition and facilitated supports being arranged for the placement before it commenced. This enhanced the likelihood that a placement will work long-term, provided a better opportunity to make a good match, and gave children time to adjust. Difficulties associated with pre-placement planning were having insufficient time to do it properly, not having enough placements, difficulty gaining information about a child, and consequently the matching process not necessarily being accurate.

Activity domains

School. Of the 17 children whom participants discussed, six had a school change, eight remained at the same school, two commenced some form of education when commencing the new placement and one was not attending any type of education institution. For those children who experienced a school change, for most this was due to a change of geographical location when changing placement. For others, this resulted from the child requesting the change or the former school not being willing to have the child return. Some participants clearly prioritised school stability and understood the contribution a stable school environment could provide.

Extracurricula activities. Few children were involved in extracurricular activities at school. Fourteen had not been participating in anything, two changed activities when they changed placements and for one child the case worker did not know if the child had been involved in anything based at the school. A similar picture also occurred in relation to extracurricular activities that the children participated outside of the school. Ten of the children were not involved in any social or extra-curricular activities; five either changed their activities or continued their activities but at a different location when moving placements and two continued with the same activities in the same location. The reasons for changing activities was at times, related to what the child wanted.

This young person wasn't really into girl guides and dance classes. At her last placement the carer and another child placed in that placement were into girl guides and dance. She did these activities because of their involvement - it was really opportunistic involvement rather than what she really wanted to do"(\#5)

Also as one participant recounted, one of the children (three year old) had not been attending a specialised program at the former placement, but a change had occurred by ensuring that the child now did attend the specialised program.

Employment. Most young people discussed did not have part-time employment. One young person had a job but when he changed placement and geographical area he was apparently not motivated to look for work again.

Contact. Following a placement change, for many of the children some level of change occurred in relation to their contact arrangements. For seven of the children there were substantive changes to their contact arrangements, for six children some elements of their contact arrangements stayed the same, whilst other aspects did change. For two children their contact remained the same. Contact arrangements for one child prior to placement were unknown as he/she had been residing with his/her parents, and one child was not having contact with anyone. There were various reasons for contact change. For a number of the children it was due to their siblings remaining in the former placement so contact was established with them. Some children wanted to re-establish contact with a parent that they previously had not wanted contact with. One young person wanted less contact when her placement changed due to strained family relations. 
Religious and cultural activities. Most of the children were not involved in any religious or cultural activities, or the case manager did not know about these $(n=10)$. Three children did experience continuity with religious and cultural activities; one experienced a change and for three children the case manager was unsure or this information was unknown.

Therapeutic activities. For the 10 children receiving therapeutic services, five children experienced a change in services, five had some services remain the same but others were changed or added.

Household routine. Most of the children experienced a change in household routine when they changed placement $(n=9)$. For seven of the children their routine remained relatively similar (there were some minor changes) and for one child this information was unknown. For those children who did experience a routine change, many believed that this was for the benefit of the child. They explained that in the children's previous placements the routine may have been nonexistent or inadequate for the needs of the child.

Personal possessions and significant objects. For most of the children who were transitioning from one placement to another, all of their possessions were taken to their new placement $(n=15)$. For the two children for whom all possessions were not transferred over, participants indicated that there were difficulties with former carers over surrendering the possessions. The following excerpt highlights this difficulty and the case manager's lack of options to address this situation. Yes she did have some possessions that were important to her but when she moved the carer was difficult to deal with about these possessions and beyond getting the sheriff in we couldn't get them for her (\#7). Some of the children did not have many personal possessions or special objects. This appeared to relate to a lack of stability in their placement and potentially grief, loss and attachment issues.

Six of the children did not have any photographs or other significant memorabilia. Seven children did have certificates and photos which were taken with them into their new placement. As with personal possessions, a few children $(n=3)$ were not able to take all of these significant pieces because of difficulties obtaining them from former carers.

\section{Discussion}

The aim of this study was to explore pre-placement planning with particular attention to the types of continuity and connections available through participation in activities. Based upon the findings, a schema for pre-placement planning is set out in Figure 1.

Insert Figure 1 here

It is recognised that the school environment can be beneficial to traumatised children especially if they have the opportunity to have continuous positive relationships with school personnel and be in an environment that is stable and predictable (Gilligan, 1998; Ferguson \& Wolkow, 2012; Fernandez, 2008). A number of the children were required to change school due to a placement change outside of their previous geographical area. Although for small number of children this was a conscious choice so they could access a placement that may have a better suitability, for others it was a discontinuity that was not necessarily desired. Also when considering these children's extracurricular activities within and outside the school a less than ideal situation was revealed. Many of the children were not involved in any leisure activities. The positive effects of children's participation in activities include: preventing placement disruption, education success, improved interpersonal competence and self-esteem (Mahoney, Cairns, Farmer, 2003 cited in Fong et al., 2006). A similar picture was also portrayed when considering religious and cultural activities - there was minimal involvement. For some children spirituality, cultural connections, and belief systems are important mechanisms for assisting them 
to cope and recover. Inquiry into the cultural activities that children are or wish to be involved in should be a part of planning (Jackson et al., 2010). These findings signal the need to assess the nature and level of support in children's wider social systems. Some of these children were not involved in anything that could be experienced as enjoyable, confidence-building and satisfying, which leads to significant gaps in their avenues for support, personal growth and wellbeing. It also has implications for identity development as discontinuities in home, culture, education, relationships and belongings can undermine the search in childhood and adolescence to gain a persistent sense of self (Ward, 2011). Deliberate and concerted connection or continuity planning by practitioners may be required. This acknowledges children's interests and every-day activities, recognising them as meaning-making actions that are important (to them), and facilitates their sense of control.

Although personal possessions generally moved along with the child to the new placement, some children had minimal personal possessions. Yet these can be important sources of personal history and identity, a tangible and concrete thread that contributes in part to a sense of security and stability (Ward, 2011). Given that some children may experience difficulties obtaining their possessions when a placement change occurs, it would appear imperative that steps be taken to clearly outline expectations and the importance of this with carers. For example, a number of the case managers in this project spoke about how they use a possession inventory which is completed for each child when a placement commences and regularly updated during a placement. This could be a good strategy for clearly stating to both the child and carer the status and expectations concerning children's possessions. For children who have minimal possessions, every effort should be undertaken to help them acquire meaningful belongings and to address the effects of disruption, including feelings of loss and grief associated with changing placement.

Discontinuities and starting again

Aiming to keep a child's life stable and continuous as possible is generally regarded as desirable, however, the findings from this project also highlight that discernment about continuity may be required. Some changes are positive. Certainly, some of the reasons for changing placement and school are not desirable, but brought about from lack of alternative options. But other changes may be purposefully planned for, such as ceasing a relationship with a former carer if it had not been positive; stopping a child undertaking risky behaviour; moving away from unhealthy peer relationships; and moving school if the school was not supportive. Children themselves may request a change in some aspects of their lives and it may be beneficial to make changes if the current situation is not positive or does not meet the individualised needs of a child. There were some children who sought a different placement or a new school so they could make a 'fresh start' and not be hindered by negative perceptions that others had of them. On the other hand, children who have to change activities and routines in their lives repeatedly may experience diminished motivation and energy to have another go. One example of this was the young person who had a part-time job at his previous placement but did not have the inclination or willpower to try again when changing placement. This may be a type of 'change fatigue', characterised by tuning out and losing energy to embrace new initiatives or opportunities (Beaudan, 2006). Rationales for discontinuity must be sound, because even planned change can be unsettling, and may have negative unintended consequences. These nuances in thinking about continuity adds weight to the imperative of talking with children, in an age appropriate manner, about what are the things that are really important to them to continue, what if anything may need to discontinue for good reason and what needs to be put on hold while 
settling occurs. Supports and strategies to facilitate continuity and the establishment of new connections will also need to be discussed and determined. Taking the time to engage with children about their ideas for placement is both a critical starting point when exploring placement options and a tangible demonstration of how children's views and involvement are paramount to placement planning. Such inclusion can be empowering and further develop their capacities (Pölkki et al., 2012).

Overall, practitioners aimed to take account of a range of needs and interests that children have when trying to identify a potential placement, and recognised the value of a smooth and planned process to give both the child and the carer time to consider, settle and adjust to a new placement. This was understood as an ideal process, but unfortunately for some placements, not able to be done due to the immediacy of the placement need and the shortage of carers. This highlights the continued need to recruit, support and successfully retain suitable carers so placement matching can be optimised (Norgate et al., 2012).

This was a qualitative study that involved semi-structured interviews with case managers from a non-government organisation who were involved in the placement of children, and because they may not be representative of all workers who are involved in the placement of children, results cannot be generalised. It is acknowledged that participants may have tried to describe their practice in the best possible way, avoiding discussion of the limitations of either their practice or the employing agency. However, they were frank about the impact of time constraints and the limits of placement choice. Insights were generated about the elements of best practice in pre-placement planning that incorporates a comprehensive conceptualisation of stability. One clear message is that continuity and connections should be an integral part of preplacement planning and considered in a deliberate and systematic manner, even if the best match cannot always be found. Ensuring that connections and activities in a child's life are continued provides the opportunity for children to have aspects of their life that are predictable and stable, which enhances the likelihood of child wellbeing. The research confirms previous findings that placements have a better chance of success if pre-placement planning occurs and a transition process is employed. Staff and carers should have training and supervision so as to raise awareness of what good placement planning entails, how to ensure good transitions, and maximise continuity and connections. This should provide a broad and comprehensive conceptualisation of stability to frame decisions about a good match, which encompasses connections to culture, activities, possessions, and known places via placement, school, relationships with family members and other people, participation in hobbies and leisure pursuits, and any other activities that encourage a 'line of continuity' for children's identity development. 


\section{References}

Australian Institute of Health and Welfare (2013). Child Protection Australia: 2011-2012. Child Welfare series no. 55. Cat. no. CWS 43. Canberra: AIHW.

Beaudan, E. (2006). Making change last: how to get beyond change fatigue. Ivey Business Journal, January/February, 1-7.

Berridge, D. (2000). Placement Stability. Quality Protects Research Briefings, 2. Retrieved from www.rip.org.uk/.

Braun V \& Clarke V. (2006) Using thematic analysis in psychology. Qualitative Research in Psychology. 3:77-101

Brown, J.D., \& Campbell, M. (2007). Foster parent perceptions of placement success. Children and Youth Services Review, 29,1010-1020.

Cashmore, J., \& Paxman, M. (1996). Wards Leaving Care - A Longitudinal Study. New South Wales: University of New South Wales and New South Wales Department of Community Services.

Cashmore, J., \& Paxton, M. (2006). Predicting aftercare outcomes: the importance of 'felt' security. Child and Family Social Work, 11,232-241.

Dudley, J.R. (2011). Research Methods for Social Work. Boston: Allyn \& Bacon.

Ferguson, H.B., \& Wolkow, K. (2012). Educating children and youth in care: A review of barriers to school progress and strategies for change. Children and Youth Services Review, 34,1143-1149.

Fernandez, E. (2008). Unravelling emotional, behavioural and educational outcomes in a longitudinal study of children in foster care. British Journal of Social Work, 38,12831301.

Ficaro, R.C. (1999). The many losses of children in substance-disordered families. In: Webb NB (ed) Play Therapy with Children in Crisis (pp. 294-317),. Second edition, New York: The Guilford Press.

Fong, R., Schwab, J., \& Amour, M. (2006). Continuity of activities and child well-being for foster care youth. Children and Youth Services Review, 28,1359-1374.

Fowler, H.W., \& Fowler, F.G. (1964). The Concise Oxford Dictionary of Current English. Fifth Edition, Oxford: The Clarendon Press.

Gilbertson, R., \& Barber, J. (2003). Breakdown of foster care placement: carer perspectives and system factors. Australian Social Work, 56(4),329-339.

Gilligan, R. (1998) The importance of schools and teachers in child welfare. Child and Family Social Work, 3,13-25.

Gilligan, R. (2008), Promoting resilience in young people in long-term care - the relevance of roles and relationships in the domains of recreation and work. Journal of Social Work Practice, 22(1),37-50.

Happer, H. McCreadie, J., \& Aldgate, J. (2006). Celebrating Success: What Helps Looked After Children Succeed. Edinburgh: Social Work Inspection Agency.

Howe, E, Dooley, T., \& Hinings, D. (2000). Assessment and decision-making in a case of child neglect and abuse using an attachment perspective. Child and Family Social Work, 5,143155.

Jack, G. (2010). Place matters: the significance of place attachments for child wellbeing. British Journal of Social Work, 40,755-771. 
Jackson, L.J., White, C.R., O’Brien, K., DiLorenzo, P., Cathcart, E., Wolf, M., Bruskas, D., Pecora, P.J., Nix-Early, V., \& Cabrera, J. (2010). Exploring spirituality among youth in foster care: findings from the Casey Field Office Mental Health Study. Child and Family Social Work, 15,107-117.

Jackson, S. (2002). Promoting stability and continuity in care away from home. In: D. McNeish, T. Newman \& H. Roberts (Eds) What Works for Children: Effective Services for Children and Families (pp. 37-58), Buckingham: Open University Press.

Miles, M. \& Huberman, A. (1994). Qualitative Data Analysis: An Expanded Sourcebook. Thousand Oaks: Sage Publications.

Mudaly, N. \& Tucci, J. (2002). Child abuse and family violence: The losses for children. Grief Matters, Summer, 43-46.

Norgate, R,, Warhurst, A., Hayden, C., Osborne, C., \& Traill, M. (2012). Social workers’ perspectives on the placement instability of looked after children. Adoption \& Fostering, 36(2), 4-18.

Pölkki, P,,Vornanen, R., Pursiainen, M., \& Riikonen, M. (2012). Children's participation in child-protection processes as experienced by foster children and social workers. Child Care in Practice, 18(2), 107-125.

Poso, T., \& Laakso, R. (2014) Matching children and substitute homes: some theoretical and empirical notions. Child and Family Social Work, early online DOI: 10.1111/cfs.12144.

Sellick, C., \& Thoburn, J. (1996) What Works in Family Placement? London: Barnardos,

Spagnola, M., \& Fiese, B.H. (2007). Family routines and rituals: A context for development in the lives of young children. Infant \& Young Children, 20(4),284-299.

Steering Committee for the Review of Government Service Provision. (2013). Report on Government Services 2013. Melbourne: Productivity Commission.

Triseliotis, J., Borland, M., \& Hill, M. (2000). Delivering Foster Care. London: BAAF. Van Nijnatten, C. (2010). Children's Agency, Children's Welfare. Bristol: The Policy Press.

Waterhouse, S., \& Brocklesby, E. (2001). Placement choice in temporary foster care. Adoption and Fostering, 25(3), 39-46.

Ward, H. (2009), Patterns of instability: Moves within the care system, their reasons, contexts and consequences. Children and Youth Services Review, 31,1113-1118.

Ward. H. (2011), Continuities and discontinuities: Issues concerning the establishment of a persistent sense of self amongst care leavers. Children and Youth Services Review, 33, 2512-2518.

Webb, N.B. (1999). Assessment of the child in crisis. In: N. B. Webb (ed) Play Therapy With Children In Crisis (pp. 3-28) Second edition. New York: The Guilford Press. 
Table 1: Practice elements

Assess the child's needs

- Develop a profile on the child (cultural background, interests, activities, etc.)

- Explore with the child perceptions of an ideal placement (what they like, what suits them)

- Examine what supports the child needs for educational and emotional wellbeing

- Gain information from other professionals involved with the child

Explore why child cannot stay in previous placement

Involve an intake panel or care team meeting

- Consult with statutory child protection worker

- Identify the skill set and approach required in the carer that matches the child's needs

Aim for a placement in the same geographical area so school, recreation, networks and friends can be maintained

Gain relevant information from previous carer to be communicated to new carers

Examine how to support the new carer and add new connections for the child if necessary

Obtain feedback from all parties about the match 
Figure 1: Framework for stability, connections and continuity planning

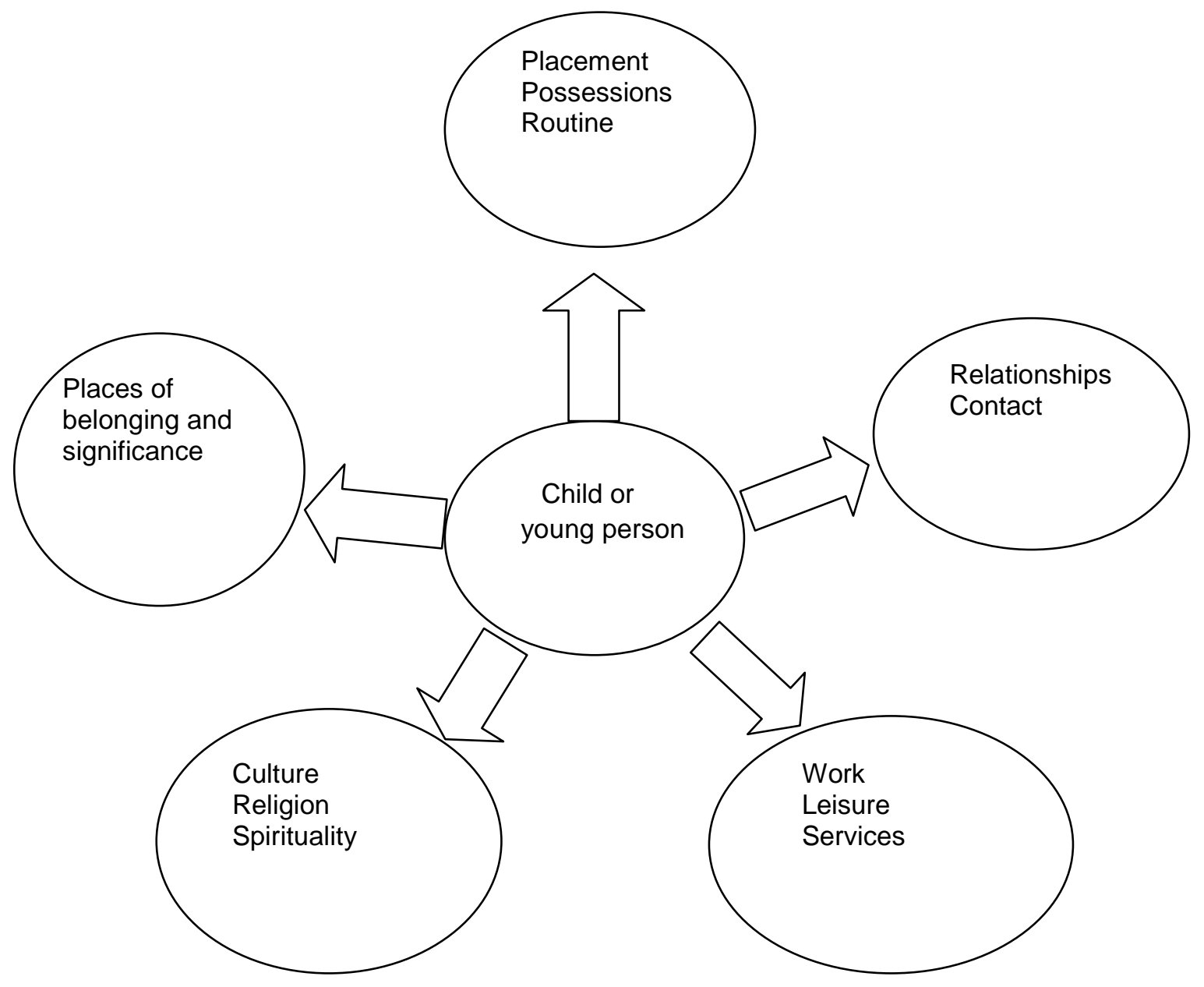

$\begin{aligned} & \longrightarrow \text { Denotes stability, connections and continuity } \\ & \text { Assess: Continue, discontinue or establish }\end{aligned}$

